\title{
基于分子马达运行机制的骨骼肌生物力学原理研究进展
}

殷跃红, 郭朝, 陈幸, 范渊杰

上海交通大学机器人研究所, 机械系统与振动国家重点实验室, 上海 200240

E-mail: yhyin@sjtu.edu.cn

2012-03-19 收稿, 2012-05-23 接受

国家自然科学基金(61075101, 60643002)、国家重点基础研究发展计划(2011CB013203)、上海交通大学理工交叉研究基金重点项目 (LG2011ZD 106 )和医工交叉研究基金重大项目(YG2010ZD 101 )资助

摘要骨骼肌是人体运动之源, 一直吸引着国内外学者竞相研究, 旨在揭示骨骼肌收缩机理, 并且相关研究成果已经获得诺贝尔奖. 本文首先阐述了骨骼肌生物力学模型研究现状, 归纳 出自上而下和自下而上的两种开展骨骼肌生物力学模型的研究策略; 并从分子马达的多力场 耦合机理与集体运行机制、骨骼肌收缩的生物电化学驱动与控制原理两大方面进行了综述, 讨论了进行实验验证的解决方案, 总结出基于骨骼肌收缩源头——分子马达的微观运行机制 来研究骨骼肌生物力学原理的新思路. 最后简要评述了目前研究中存在的不足, 探讨了今后 需深入研究的方向.

关键词

骨骼肌

分子马达

生物力学模型

肌小节

运行机制
骨骼肌通过肌腱与人体关节相连, 在神经系统 的支配下产生收缩并驱动关节运动. 人体有六百多 块骨骼肌, 约占人体体重的 $40 \%$. 因其对运动的重要 作用, 骨骼肌一直是国内外学者竞相研究的热点之 一, 旨在揭示骨骼肌收缩的生物力学原理. 最早取得 突破性进展的是诺贝尔奖获得者 $\mathrm{Hill}^{[1]}$ 和 Huxley $^{[2,3]}$ 的研究工作, 他们的成果奠定了骨骼肌生物力学原 理研究的基础. 后来的研究大多是在此基础上, 分别 从骨骼肌结构和功能等方面展开 ${ }^{[4]}$. 特别是近年来微 纳技术的快速发展, 骨骼肌生物力学原理研究成为 了生物力学中的一大重点, 主要通过揭示骨骼肌的 力学特性构建相应的力学模型, 并开展模型在运动 生物力学、仿生学、康复工程等方面的应用研究. 从 宏观上来看, 骨骼肌具有主动收缩和被动拉伸特性. 骨骼肌的被动拉伸性质与许多生物软组织相似, 目 前已有多种模型进行描述 ${ }^{[5]}$. 但是, 与软组织不同的 是, 骨骼肌可以在神经系统的控制下主动收缩, 这也 是骨骼肌生物力学原理研究中的重点与难点. 从微 观上来看, 由于骨骼肌收缩过程涉及神经系统控制、 动作电位传递、钙离子扩散、分子马达做功、肌小节 收缩等步骤, 其力学原理研究十分复杂, 难度较大,
但由于其重要的应用价值, 仍吸引着众多学者在此 领域进行探索.

本文总结了近年来从微观角度开展骨骼肌生物 力学原理研究的现状, 结合国内外的相关工作, 分别 从骨骼肌解剖学结构、生物力学模型、微观收缩机理 及生物电化学驱动与控制原理几个方面, 对骨骼肌 生物力学原理的研究进展进行了综述, 探讨目前研 究中存在的不足以及今后需深人研究的方向.

\section{1 骨骼肌解剖学结构}

骨骼肌的生理结构如图 1 所示, 从宏观上来看, 骨骼肌由肌原纤维(myofibril)并行排列而成的肌纤维 组成(图 1(a)), 肌肉上还有结缔组织、本体感受器、 运动神经元等其他组织. 从介观上来看, 肌原纤维呈 细丝状(图 1(b)); 肌小节(sarcomere)是肌肉收缩的最 小单位, 它沿轴向串联组成肌原纤维. 从微观上来看, 肌小节主要包含粗肌丝和细肌丝, 细肌丝由肌动蛋 白(actin)、原肌球蛋白(tropomyosin, Tm) 和肌钻蛋白 (troponin, Tn) 构成(图 1(c)), 粗肌丝由肌球蛋白 (myosin II)组合而成(图 1(d)), 连接粗肌丝与 Z 线的 是具有弹性的肌联蛋白 ${ }^{[6]}$. 肌球蛋白是一种线性(动)

英文版见: Yin Y H, Guo Z, Chen X, et al. Studies on biomechanics of skeletal muscle based on the working mechanism of myosin motors: An overview. Chin Sci Bull, 2012, 57, doi: 10.1007/s11434-012-5438-y 


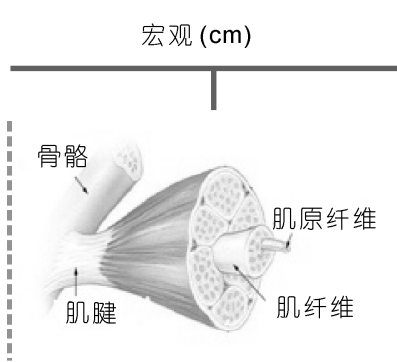

(a)

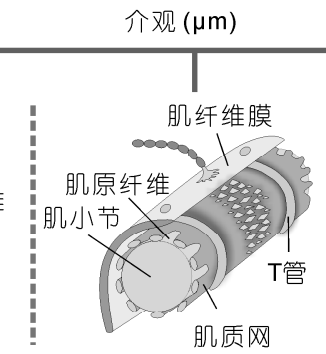

(b)

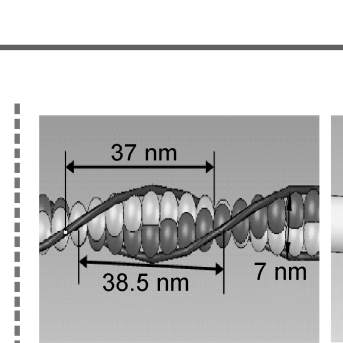

(c)
微观 $(\mathrm{nm})$

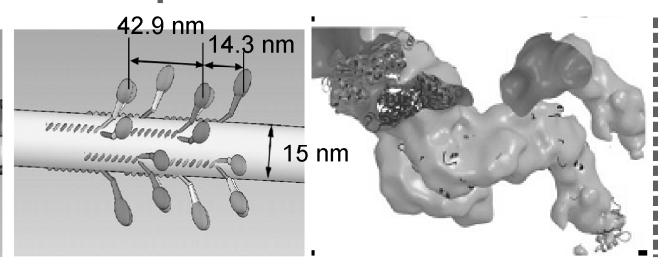

(d)

(e)

图 1 骨骼肌各层次结构组成

(a) 骨骼肌; (b) 肌原纤维; (c) 细肌丝; (d) 粗肌丝; (e) 肌球蛋白头部

分子马达, 它通过集体牵拉细肌丝做功引起肌小节 收缩. 1993 年 Rayment 等人 ${ }^{[7,8]}$ 利用 X 射线衍射技术 确定了肌球蛋白的微观结构, 其可分为三个部分：头 部、颈部和尾部. 头部具有肌动蛋白结合部位及 ATP 结合部位, 相互作用力产生于头部的肌动蛋白结合 区, 头部也称为横桥 (cross bridge); 与头部相连的颈 部是 $\alpha$-螺旋, 它结合着两个轻链(light chain), 颈部起 着调整头部活动的作用 ${ }^{[9]}$. Holmes 等人 ${ }^{[10]}$ 利用扫描 电子显微镜观测了肌球蛋白三维结构及与肌动蛋白 结合过程(图 1(e)).

\section{2 骨骼肌生物力学模型及其研究策略}

骨骼肌生物力学模型研究属于基础生命科学研 究之一 ${ }^{[11,12]}$, 源自 Hill 的开拓性工作. 1938 年 Hill $^{[1]}$ 通过青蛙骨骼肌快速释放实验推导出 Hill方程，该方 程描述了肌肉力与收缩速度之间关系, 但它只反映 了肌肉一个方面的性质. 后来, 为了完整描述肌肉宏 观性质, Hill 提出了三元素模型, 即肌肉由非线性收 缩元、串联弹性元和并联弹性元组成. Hill 模型建立 在离体肌肉实验结果的基础上，反映了骨骼肌的宏 观属性, 其最大不足是简化了很多环节, 存在精度不 高的缺点. 为此很多学者对 Hill 模型进行了修正, Zajac 等人 ${ }^{[13]}$ 考虑了肌腱在骨骼肌力传递中的作用, 将其作为串联单元和肌肉连接, 修正了 Hill 模型. Hill 模型及其修正模型描述了肌肉宏观力学性质, 它 的提出奠定了骨骼肌宏观生物力学研究的基础, 但 由于技术原因，没有考虑肌肉收缩的微观特性，尚无 法有效地反映活体肌肉的力学性质, 使得其在实际 应用中存在不足。

随着显微技术的发展, 研究人员可以逐渐观测 到骨骼肌微观结构, 骨骼肌力学原理研究也从宏观
深人到微观. 根据显微镜的实验观察结果, Huxley 等 人提出 “肌丝滑行理论”, ${ }^{214,15]}$, 认为收缩是通过细肌 丝和粗肌丝之间的滑行实现，这一理论的提出对骨 骼肌研究贡献很大. 接着, 为了进一步描述肌肉微观 力学性质, Huxley ${ }^{[16]}$ 提出了横桥动力学模型 (crossbridge model), 并通过假设给出了横桥结合速率方程. Huxley 模型奠定了骨骼肌微观力学原理研究的基础, 具有十分重要的意义. 1966 年 Gordon 等人 ${ }^{[17]}$ 通过实 验研究了单根肌纤维收缩力与长度的关系, 研究表 明在一定范围内, 收缩力与细肌丝与粗肌丝之间的 重叠长度成正比, 当处于最适长度时肌力达到最大 值, 继续拉伸由于重叠程度的减小, 肌力也逐渐减小 到零. 这一点与 Hill模型描述肌肉力学与长度关系一 致, 本质上由肌纤维的对称性结构导致. 后来, Zahalak 等人 ${ }^{[18]}$ 将 $\mathrm{Ca}^{2+}$ 激活细肌丝的过程引人到 Huxley 模型中，对速率方程进行了修正. Huxley 模型 及其修正模型是以骨骼肌微观结构为基础的模型, 它建立在分子马达与细肌丝结合与分离循环假设基 础上, 推导出肌肉力公式, 但是该模型没有建立肌肉 微观与宏观之间的联系. 特别需要指出的是, 2002 年 Piazzesi 等人 ${ }^{[19]}$ 采用一种新的 X 射线技术测量马达头 部轴向运动, 研究结果表明分子马达的做功冲程 (power stroke)模型能更好地解释骨骼肌收缩力产生 原理，而不是 Huxley 所采用的“结合与分离假说”.

由此可见，虽然 Huxley 提出了基于骨骼肌微观 原理的建模思想, 但是对于分子马达微观循环过程 缺少更加具体的描述，也并未完成骨骼肌收缩微观 与宏观力学特性的有效统一. 随着纳米技术的快速 发展，分子层面的骨骼肌结构已可以被观测，而原子 力显微镜 $(\mathrm{AFM})$ 、光镊等单分子操纵技术的应用, 分 子马达与细肌丝间的相互作用力也被检测出来 ${ }^{[20]}$, 
因此可以深人分析分子马达循环运行机制, 并在此 基础上根据非平衡态统计力学方法研究分子马达集 体运行机制, 建立肌小节力学模型, 然后由肌小节的 串联与并联特征, 构建骨骼肌宏观的动态力学模型. 这种方式是一种从微观到宏观自下而上探索骨骼肌 生物力学原理的研究策略.

对于骨骼肌生物力学模型的验证, 从现有研究 成果来看, 主要有两种途径: 一种是人体运动逆向动 力学(inverse dynamics)方法, 该方法是已知人体运动 和作用力信息求解单块肌肉的动态作用力进行验证. 人体运动信息, 如关节的角度、运动速度和作用力可 由步态分析系统等实验设备测量, 通过建立人体关 节系统动力学模型, 反求出作用在各关节上力矩, 并 由解剖学已知关节力臂的大小, 采用最优化约束方 法可求出相应肌肉力 ${ }^{[21]}$. 但是利用逆动力学方程求 解肌肉力时存在肌肉力元余问题, 制约了其进一步 的推广运用. 另一种方法是人体运动正向动力学 (forward dynamics), 它为肌肉力大小的计算提供了 新的途径. 该方法是基于肌肉力学模型建立从神经 信号到关节运动各个过程的数学模型来研究人体运 动. 例如 Neptune 等人 ${ }^{[22]}$ 利用正向动力学分析手段, 建立 17 块基于 Hill 模型的肌肉来分析人体下肢步态 运动. 值得一提的是, 杨义勇等人 ${ }^{[23]}$ 以下肢步态为 研究对象, 建立了包含神经兴奋-肌肉收缩动力响应 和肌肉-肌腱动力特性在内的下肢系统正向动力学模 型, 并针对自然步态的运动过程, 采用参数最优化方 法进行了模拟计算; 由于采用了 Hill 模型, 计算结果 的精度有限.

需要指出的是, 人体骨骼肌生物力学模型的实 验验证一直存在困难. 现有的模型大多立足于假设 或者动物肌肉离体实验, 难以保证与活体肌肉性质 完全一致. 然而直接通过提取人体活体肌肉进行实 验肯定是不可能的, 但实验验证又是进行骨骼肌生 物力学模型研究必不可少的核心内容, 那么一个问 题自然而然的产生: 能否用其他方法解决这一瓶颈 难题? 答案是肯定的. 可利用肌电仪、运动捕捉系 统、测力平台等实验设备, 结合解剖学知识, 结合正 向动力学与逆向动力学两种方法优点, 进行人体运 动生物力学分析, 获取肌肉动态力学信息来进行验证. 由于人体运动的传递过程: 神经信号-肌肉力-关节运 动, 肌纤维上的动作电位在皮肤表面叠加形成表面肌 电信号(surface electromyography signal, sEMG), sEMG
信号能够表征肌肉的收缩力、收缩速度等动态特性, 可通过贴在皮肤上的电极检测出来, 在关节运动时, 肌电信号的强弱可用来表征参与运动肌肉的激活水 平. 因此, 可以通过先进仪器采集人体运动和力信息, 进行人体逆向动力学计算, 求出关节力矩作为验证 数据. 并利用从微观上构建的骨骼肌生物力学模型, 结合 sEMG 信号表征肌肉激活程度, 结合解剖学数 据, 展开人体正向动力学分析求取关节力矩, 然后与 逆向动力学所求得的力学数据相比较, 判断和验证 所建立模型的准确性. 所以, 与自下而上的研究方式 相对应, 这是另一种从宏观到微观自上而下验证骨 骼肌生物力学原理的重要途径.

综上所述, 首先在研究肌肉收缩的微观动态过 程基础上自下而上建立骨骼肌生物力学模型, 再进 行自上而下的实验验证研究来解决微观力学行为与 宏观力学模型的统一性问题, 是开展骨骼肌生物力 学原理研究的有效途径. 而其中最为关键的是揭示 骨骼肌收缩微观机理及驱动与控制原理, 因此需要 深人到骨骼肌微观层次, 分析肌球蛋白与肌动蛋白 从接近、接触到相对运动循环过程中的多力场耦合机 理及运行机制, 研究分子马达的集体特性、骨骼肌收 缩生物电化学驱动与控制原理, 探讨由肌小节串并 联构成的骨骼肌宏观力学特性, 建立骨骼肌生物力 学模型.

\section{3 骨骼肌收缩的微观机理}

如前所述, 骨骼肌收缩是肌球蛋白分子马达在 细肌丝上集体做功引起, 所谓分子马达是指一类将 化学能直接转化为机械功的分子机器, 它们在生命 体内起重要作用, 主要通过利用三磷酸腺苷(ATP)水 解所释放的化学能完成各种运动 ${ }^{[24]}$. 肌肉收缩力起 源于肌球蛋白和肌动蛋白之间的交互作用过程. 首 先肌纤维上的动作电位引起肌质网(SR)上的 $\mathrm{Ca}^{2+}$ 通 道开启, $\mathrm{Ca}^{2+}$ 扩散进人肌浆并使其 $\mathrm{Ca}^{2+}$ 浓度升高, 紧 接着 $\mathrm{Tn}$ 与 $\mathrm{Ca}^{2+}$ 结合后引发 $\mathrm{Tm}$ 构象改变, 露出肌球 蛋白结合点位 ${ }^{[25]}$, 分子马达通过与细肌丝强结合、水 解 ATP、发生构象改变形成一个做功冲程 ${ }^{[26]}$, 此循环 过程称为 Lymn-Taylor 循环 ${ }^{[27]}$; 分子马达集体做功 引起粗肌丝和细肌丝的相对滑动, 使肌肉产生收缩. 揭示骨骼肌收缩微观机理的研究主要包括以下几个 方面: (1) 对分子马达运作的力场环境的分析, 此类 研究揭示马达在多种微力耦合作用下的运行机理; 
（2）从分子构象变化原理与布朗棘轮理论这两个角 度出发, 对单个分子马达循环运行机制的解释; (3) 对分子马达集体运行机制的研究, 此类研究是骨骼 肌收缩理论从微观到宏观的过渡, 可帮助建立骨骼 肌收缩的力学模型.

\section{1 分子马达多力场耦合}

目前对于肌肉收缩的微观机理研究主要集中在 过程分析上, 关于肌球蛋白与肌动蛋白结合的动态 过程(包括相互作用力的产生原理和动力学行为)的 研究较少. 从肌球蛋白马达与肌动蛋白相互作用的 接近距离来看, 接近过程中的作用力应该为静电力、 van der Waals 力、 Casimir 力、水合力和疏水作用力 等, 每一种力都有其作用条件和作用范围 ${ }^{[28]}$. 其中 van der Waals 力和 Casimir 力很类似, 在真空中当粒 子之间的间距 $5 \mathrm{~nm} 1 \mu \mathrm{m}$ 时 Casimir 力起主导作用, 一般认为在间距小于 $5 \mathrm{~nm}$ 时, 起主导作用的是 van der Waals 力, 而在液体介质中该距离会更短. 分子 马达尺寸只有十几纳米, 处于 van der Waals 力和 Casimir 力的过渡范围. 值得一提的是, 2009 年 Federico 等人 ${ }^{[29]}$ 首次测量出排斥性 Casimir 力, 并指 出排斥性 Casimir 力能让物体在纳米尺度上悬浮于液 体中, Casimir 力在液体中得到实验证实, 为研究多力 场耦合问题时引人 Casimir 力提供了有力的支持. 相 比较而言, van der Waals 力是一种分子力, 对于肌球 蛋白和肌动蛋白大分子，它们之间的 van der Waals 力也是很重要的影响因素. 另外 Rayment 等人 ${ }^{[7,8]}$ 通 过观测肌球蛋白的分子结构发现, 其头部存在带负 电的分子基团，而肌动蛋白结合位点为带正电荷基 才，它们之间的静电引力可使彼此接近. Liu 等人 ${ }^{[30]}$ 通过分子动力学模拟的方法分析肌球蛋白与肌动蛋
白的相互作用, 发现静电吸引力是 van der Waals 力 的 4 倍, 在吸引力中占主导地位. 在肌球蛋白与肌动 蛋白相互作用力大小和位移的测量研究上也取得了 新进展. 最具代表性的是 Finer 等人 ${ }^{[20]}$ 利用光镊技术 测量了肌球蛋白沿着微丝运动的距离和相互作用力, 实验结果表明一个 ATP 水解产生的能量, 可使肌球 蛋白沿肌动蛋白轨道以 5 10 $\mathrm{nm}$ 的步伐前进, 马达产 生力大小在 4 6 pN 之间, 如图 2(a)所示. 同时, Nakajima 等人 ${ }^{[31]}$ 利用 $A F M$ 测量了肌球蛋白向肌动蛋 白接近过程中的静电作用力, 如图 2(b)所示, 其大小 在 $18.4 \sim 24.7 \mathrm{pN}$ 之间.

在文献[32,33]中, 我们针对肌球蛋白分子马达 的工作过程, 结合分子力学与分子检测实验的最新 研究成果, 重点探索了分子马达向肌动蛋白接近过 程中的力学原理, 揭示出肌球蛋白马达在 van der Waals 力、 Casimir 力、静电力及布朗力等多力耦合 作用下的运动规律，建立了基于两蛋白分子相互作 用势能的微观动力学模型, 并采用 Monte Carlo 方法 对动力学模型进行了模拟计算. 比较几个力的影响 可以发现, 接近过程中静电引力起主导, 这一结果与 实验测量数据吻合. 以上研究揭示了骨骼肌收缩过 程中分子马达所处微观环境遵循的力学准则及力学 原理, 为进一步开展分子马达循环运行机制的研究 提供了一定基础.

\section{2 分子马达运行机制}

(i) 分子马达循环运行机制. 目前解释分子马 达单循环运行机制主要有两类模型 ${ }^{[34,35]}$. 一类是相 对确定性的描述，即构象变化模型(conformational change model), 将分子马达产生的力与位移归因于 其内部的分子基团重组与变构. 另一类是统计热力

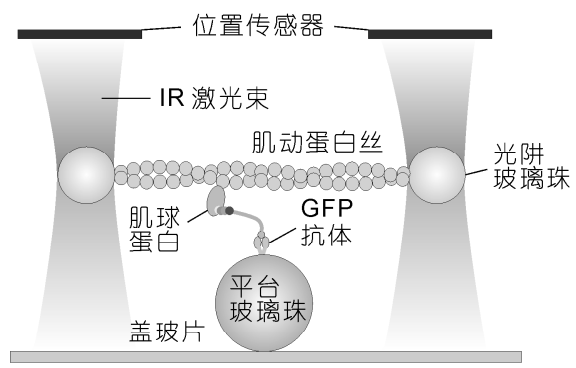

(a)

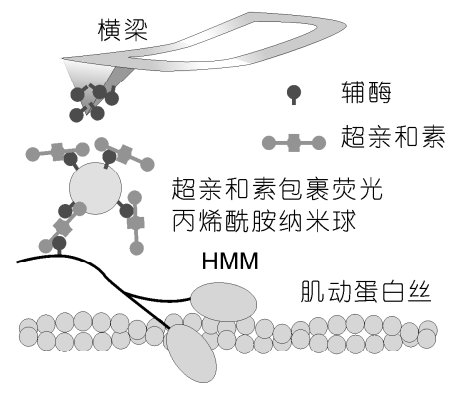

(b)

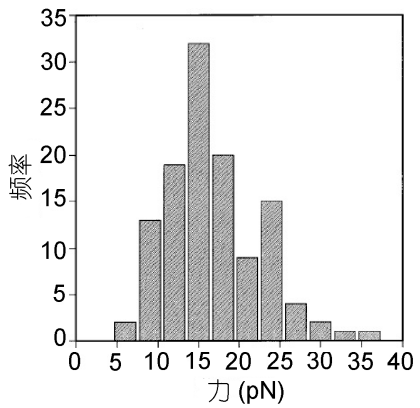

图 2 (a) 分子马达对细肌丝的推力检测 ${ }^{[21]}$; (b) 分子马达接近过程作用力检测 ${ }^{[31]}$ 
学模型, 也称非对称布朗棘轮模型(biased Brownian Ratchet model), 属于概率性描述, 将分子马达的运 动解释为在棘齿状周期势场中进行偏向性的布朗热 运动. 这两类模型对分子马达的研究各有贡献, 并且 在解释马达运行的某些特性上都获得了一定的成功. 然而两者都有各自的局限性, 并且他们之间尚存在 矛盾.

（1）构象变化模型. 最先被广泛接受的构象变 化模型是 Huxley 提出的横桥模型 ${ }^{[35]}$. 横桥是指马达 头部与细肌丝强结合之后所形成的结构. Huxley认为 这样的结构加强了肌小节的刚度, 而马达头部的转 动是力产生的源头, 并导致了粗细肌丝之间的相对 位移. 针对当时的实验现象, 横桥模型给出了初步的 理论解释, 其工作具有奠基性, 然而此模型不够细致, 只是对分子马达运行机制的定性解释. 单分子实验 一直推动着分子马达运行机制的研究进程: Kaya 等 人指出肌球蛋白马达在一个做功冲程内可前进约 8 $\mathrm{nm}^{[36]}$, 并且马达颈部的非线性弹性特征对马达产力 有显著影响, 而 Sellers 等人使用光阱技术直接观察 了分子马达做功冲程构象的可逆性 ${ }^{[37]}$. 另一方面, Spudich 在总结前人工作基础上提出了摇摆横桥模型 (swinging cross-bridge model) ${ }^{[26]}$ 或力臂模型(lever arm model $)^{[38]}$. 力臂模型是对横桥模型的细化与改进, 此 模型不仅提出了肌球蛋白头部几个环路对马达与细 肌丝及 ATP 结合的关键作用, 还确定了肌球蛋白上 马达功能区域(motor domain)的位置是在马达头部与 颈部的连接部位附近, 如图 3(a)所示. 所谓力臂是指 马达的颈部, 当 ATP 水解后马达功能区域的分子基 团发生重组与形变, 并被颈部放大, 由颈部旋转约 $70^{\circ}$ 产生数纳米的直线位移, 而力臂的刚度被颈部的 基础轻链 (ELC) 与调解轻链 (RLC) 加强. 力臂模型被 公认为第二代构象变化模型, 并沿用至今, 此后更细
致的实验工作都以此模型为蓝本. 值得一提的是, 日 本的 Osaka 小组对分子马达的单分子实验研究做出 了重要贡献, 此研究组将光镊技术与苂光显微镜结 合, 定量观测到单个 ATP 水解时, 马达前进的动态 位移与系统刚度变化 ${ }^{[39]}$, 实验原理如图 4 所示. 他们 还揭示了肌球蛋白头部与细肌丝之间的空间夹角对 于位移测量的重要影响 ${ }^{[40]}$, 并利用微探针技术观测 了分子马达的运动细节, 实验原理如图 5(a)所示, 结 果表明当马达头部处于最佳方向时, 在一个 ATP 水 解周期内, 马达可带动细肌丝前进 $15 \mathrm{~nm}$ 左右, 并且 整个过程分几个小步(substep)完成, 每个小步约为 $5.5 \mathrm{~nm}$ (图 5(b)). 他们还观测到马达位移的产生可在 ATP 水解后数毫秒内发生, 即马达可储存 ATP 水解的 能量, 而与肌动蛋白的结合开启了马达的做功循环.

构象变化模型的物理本源是分子基团或单分子 之间的反应, 涉及到量子力学 $(\mathrm{QM})$ 与分子力学 $(\mathrm{MM})$ 机制. 对于这类模型的研究多基于单分子实验或分 子动力学仿真分析, 目的在于考查肌球蛋白中哪些 基团产生了何种变化导致了马达产力与形变. 近年 来, 一种将 $\mathrm{QM}$ 与 $\mathrm{MM}$ 组合的方法 $(\mathrm{QM} / \mathrm{MM}$ hybrid method)在生物大分子酶催化反应等问题的分析中得 到应用. 该方法用 $\mathrm{QM}$ 方法计算原子级别的反应，用 $\mathrm{MM}$ 方法研究分子基团的相互作用, 这样的组合能 够在计算速度与精度之间取得最优平衡. 随着 $\mathrm{QM} / \mathrm{MM}$ 组合方法的不断发展和完善, 这种高效的计 算方法已经在研究生物大分子的结构与反应等方面 取得了成功 ${ }^{[11 ~ 43]}$, 为进一步开展分子马达运行机制 研究提供了新的思路.

(2) 布朗棘轮模型。布朗棘轮的概念最早由 Feynman 提出 ${ }^{[44]}$, 他提出了一个假想的微观棘轮模 型, 使得粒子往某个方向的扩散较容易 (势吽较缓), 而向反方向的扩散较困难(势吽较陡), 因此粒子往某

(b)

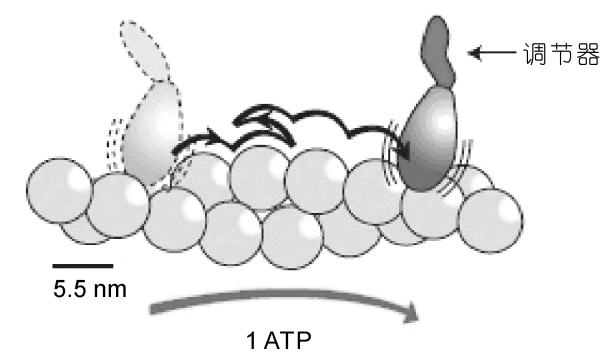

图 3 (a) 构象变化模型; (b) 布朗棘轮模型 ${ }^{[34]}$ 


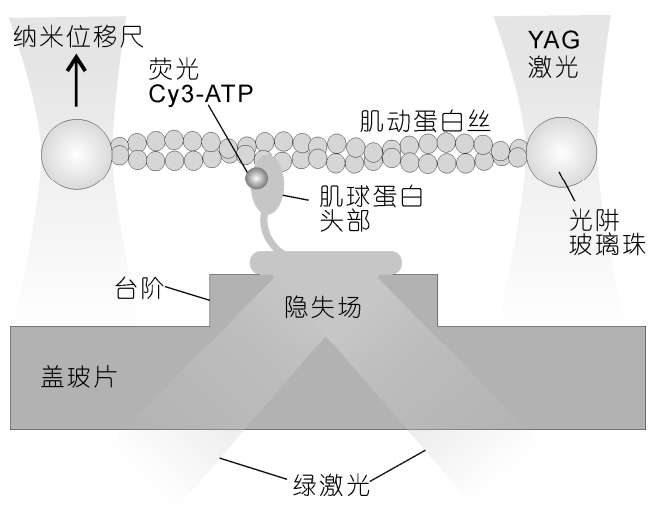

图 4 分子马达单分子荧光实验原理

个方向的扩散概率就更大, 使宏观上的物质定向输 运得以产生. 在实际的物理应用中, 此假想的棘轮与 非对称的势能分布等价, 而这类势能被称为棘轮势. 分子马达的布朗棘轮模型是指在肌动-肌球蛋白系统 中, 肌球蛋白的头部可认为是在胞浆中扩散的布朗 粒子，而周期性的棘轮势则起源于细肌丝上周期性 分布的肌球蛋白结合位点, 而对于势能不对称的成 因则存在多种观点, 例如由于马达头部结构的不对 称或 ATP 的结合等 ${ }^{[45]}$. 布朗棘轮模型认为分子马达 在棘轮势的作用下向前冲浪, 以产生力与位移, 虽然 偶尔也有后退的运动, 但从统计平均的意义上讲, 总 体效果还是前向运动, 如图 3(b)所示. 布朗棘轮模型 的核心机制由 Langevin 方程描述, 方程中的惯性力 项往往被忽略, 这是由于马达本身质量十分小, 且处 于过阻尼溶液环境. 布朗棘轮模型的具体描述有以 下几种形式 ${ }^{[46]}$ : 涨落力模型(fluctuating force)、涨落 势模型 (fluctuating potential)、多态涨落模型 (fluctuating between states). 当分子马达处于近平衡 态时, 其能量转换与粒子输运的联系多用 Onsager 关 系来表达, 定向输运发生的条件为至少存在一组跃
迁不满足细致平衡. Esaki 等人 ${ }^{[47]}$ 对分子马达的布朗 运动过程进行了模拟, 试图以此解释单分子马达的 实验现象. 在国内, 艾保全等人 ${ }^{[48]}$ 建立了一个物理 随机倾斜杆模型, 研究了肌球蛋白定向运动的原理. 包景东等人 ${ }^{[49]}$ 提出了一个研究分子马达单向梯跳运 动的含色噪声的偏压涨落模型, 用 Monte-Carlo 方法 模拟了马达分子的力与速度关系和梯跳运动, 李晨 璞等人 ${ }^{[50]}$ 利用偶极子模型研究肌球蛋白 VI 的定向 运动.

如图 5(b)所示, 在单分子实验中, 数据显示马达 消耗一个ATP 分子可移动距离约为 $15 \mathrm{~nm}$, 并且此距 离由约为 $5.5 \mathrm{~nm}$ 的小分步组成 ${ }^{[34]}$. 完成此实验的 Osaka 小组认为分步位移的大小与肌动蛋白单体 (G-actin)的间距相一致, 并由此推断马达的移动由布 朗棘轮机制造成. 然而如果在一个 ATP 的水解周期 中, 马达还可以屡次从细肌丝上脱离, 则很难想象分 子马达还能够有效运作. 另外, 单从图 5(b)的数据来 看, 马达也并未从细肌丝脱离, 所有分步基本都呈现 连续的阶跃. 因此 $5.5 \mathrm{~nm}$ 的小分步与肌动蛋白单体 间距相接近或许只是一个巧合, 而对于分子马达的 做功冲程来说，马达头部构象变化的解释看似更合 理; 则马达的小分步可能是由于 ATP 水解的能量阶 段性释放造成, 这也暗示了在肌球蛋白的分子结构 中可能存在 $4 \sim 5$ 个能级, 需要从自由能的角度结合 $\mathrm{QM} / \mathrm{MM}$ 方法分析其跃迁机制. 可见，在应用布朗棘 轮理论时, 人们过多地关注其功能性而忽略了一些 基本问题，所以应当首先把物理模型本身的物理意 义与所考虑的对象进行匹配与统一, 合理地看待棘 轮模型.

构象变化模型与布朗棘轮模型的比较如表 1 所 示. 构象变化模型有实验数据的支持, 尽管模型还有

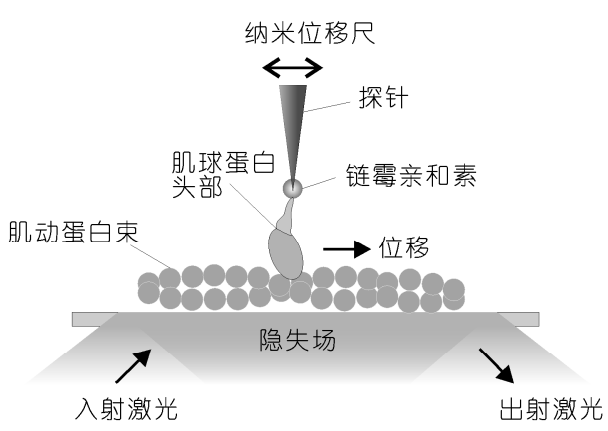

(a)

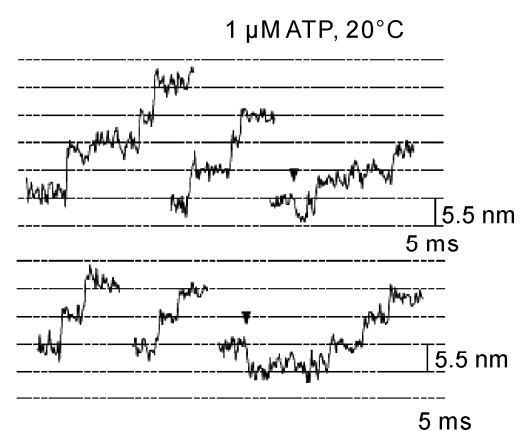

(b)

图 5 (a) 分子马达单冲程位移测量原理; (b) 单个分子马达单个做功冲程中的小分步 ${ }^{[34]}$ 
表 1 构象变化模型与布朗棘轮模型的比较

\begin{tabular}{lll}
\hline \multicolumn{1}{c}{ 性质 } & \multicolumn{1}{c}{ 构象变化模型 } \\
\hline 力学基础 & 量子力学/分子力学 & 统计热力学 \\
描述特轮模型 & 相对确定 & 相对随机 \\
分析方法 & 分子动力学仿真 & 物理建模/统计行为模拟 \\
与生物分子结构的联系 & 紧密 & 不紧密 \\
适用范围 & 肌动-肌球蛋白的结合/脱离 & 肌球蛋白脱离后的运动 \\
& 肌球蛋白的做功冲程 & 细肌丝上结合位点的选择 \\
\hline
\end{tabular}

待完善, 但对于后僵直态时肌球蛋白如何与肌动蛋 白脱离、预功冲程中 ATP 水解如何影响马达的基团 结构以及马达在做功冲程中如何产生力与位移等方 面, 其描述都是直接有效的. 然而构象变化模型未能 解释马达在一个 ATP 水解循环中为何能够行进 15 $\mathrm{nm}$ 的距离 (现有的模型预测为 5 10 nm), 以及马达位 移中的小分步如何形成, 特别是在预功冲程 (prepower stroke)阶段, 对于马达如何选择下一个结合位 点以及怎样与细肌丝接近, 此模型皆未给出合理解 释. 特别是最近, Spudich 指出对于 myosin VI 的实验 结果对摇摆横桥模型很具有挑战性, 因其在做功冲 程中超出了模型预言的运行距离 ${ }^{[51]}$; Sweeney 等人对 myosin VI 的研究表明分子马达的运行规律需要被改 写 ${ }^{[52]}$, 因此, 很可能在马达运行过程中不单是构象 变化机制在起作用. 布朗棘轮模型在描述分子马达 从肌动蛋白上脱离到与之再结合的过程时具有一定 的优势, 并且经过适当的调整, 确实可以使理论结果 与相应的分子马达实验数据相符合, 然而布朗棘轮 模型的局限性在于模型过于注重数学描述, 对分子 马达运行过程的解释过于抽象; 模型本身带有很强 的功能性, 其中势能与力的物理源头不够明确, 包括 能量曲面不对称的原因仍难以解释; 此外, 模型对肌 动-肌球蛋白系统的分子结构忽略过多, 为了解释实 验现象, 模型的众多参数都需要人为调整, 这些因素 削弱了布朗棘轮模型的有效性. 可见, 构象变化模型 与布朗棘轮模型的出发点分别是量子/分子力学机制 与统计热力学机制, 其力学基础本身不一致; 而对于 实验现象的解释, 两者各有其优势, 又有各自的局限 性. 因此, 对于分子马达运行机制研究, 关键在于对 现有模型进行统一, 即对分子马达运行中的量子力 学、分子力学以及统计热力学机制进行统一描述.

由于生物分子马达是自然界最优化的分子机器, 对分子马达运行机制的研究也推动了人工分子马达 及纳米制造技术的进步，利用分子组装方法设计并
制造出可作为药物载体、疾病诊断与治疗的生物纳米 器件, 是当前生物纳米技术研究的国际前沿领域. 如 Montemagno 研究小组 ${ }^{[53]}$ 制造出一种旋转分子马达, 这种马达以 ATP 酶分子马达为基础, 通过将金属镍 制成的螺旋桨嫁接 ATP 酶分子中轴上，制成了简易 的超微螺旋推进器. Van Delden 等人 ${ }^{[54]}$ 根据 ATP 酶 马达的工作原理, 研制了一种光驱动分子马达. 在国 内, 赵亚溥等人 ${ }^{[55]}$ 将纳米金属镍丝与 ATP 酶马达中 的 $\mathrm{F}_{1}$ 分子马达连接在一起构成纳米推进器, 同时设 计调控磁场, 实现了对旋转分子马达的控制. 崔元波 等人 ${ }^{[56]}$ 对光驱动 $\mathrm{F}_{0} \mathrm{~F}_{1}$-ATP 合酶复合物的旋转进行了 观察, Qi 等人 ${ }^{[57]}$ 利用分子组装技术将从植物中提取 的 ATP 酶马达组装到磷脂修饰的微胶囊上. 因此, 开展分子马达运行机制研究具有重要的理论意义.

(ii) 分子马达集体运行机制. 肌球蛋白是一种 非持续性马达, 具有较小的占空比, 在细肌丝轨道上 驻留时间短, 为了产生力和运动需要大量的马达协 调工作, 因此骨骼肌收缩力学特性由分子马达的协 作运动产生, 并且满足统计力学规律. 基于统计力学 原理研究分子马达的集体运行机制是探索从微观到 宏观构建骨骼肌收缩的力学模型的有效方法. 从目 前对分子马达集体运行机制的研究现状来看, 主要 从两个方面展开, 从化学动力学角度, Lan 等人 ${ }^{[58]}$ 基 于单个马达的构象变化情况构建了稳态时的肌肉力 学模型, 并以细肌丝为对象研究收缩力与速度关系. Chin 等人 ${ }^{[59]}$ 采用 7 种状态来描述分子马达循环过程, 并进一步修正了力与速度关系. 郭维生等人 ${ }^{[60]}$ 给出 一个肌球蛋白工作循环的机械化学偶联模型, 并用 一组化学动力学方程描述肌肉中大量肌球蛋白的集 体行为, 对肌肉的自发振动进行了研究. 从统计物理 学角度, Julicher 等人 ${ }^{[46]}$ 在二态棘轮模型基础上利用 统计力学方法, 建立反映马达集体特性的统计力学 方程, 分析分子马达的集体特性; Shu 等人 ${ }^{[61]}$ 基于二 态棘轮模型定性讨论集体特性对 ATP 水解动力学的 
影响, 揭示 ATP 浓度与自发振动之间关系. 物理方 法主要着眼于分子马达运行机制的定性分析，难以 反映系统的实际力学特性; 化学方法结合分子马达 化学状态的变化分析肌肉收缩机理, 但是缺乏对肌 肉收缩过程的统一数学描述, 针对集体特性的定量 计算较少.

有关分子马达集体协作机制的研究还有待深人, 需要结合化学动力学与统计物理学两种方法的优点, 建立相应的数学模型, 并分别讨论影响集体特性的

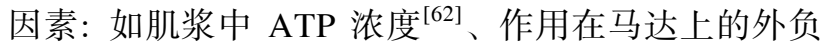
载 ${ }^{[63]}$ 、以及马达之间的作用势等的作用. Brugues 等 人 ${ }^{[64]}$ 分析分子马达在外负载作用下的集体动力学行 为, 考虑分子马达之间相互作用势影响, 并通过计算 发现外负载可以增加马达的协同性，集体运动的效 率比单个马达高; Campas 等人 ${ }^{[65]}$ 研究多个分子马达 的集体动态行为, 计算发现力与速度曲线取决于分 子马达之间的相互作用, 这一特征和单个马达作用 明显不同. 因此, 在分子马达的集体运行过程中, 必 须考虑负载力对马达集体特性的影响, 我们利用非 平衡态统计力学方法分析肌球蛋白马达的集体运行 特性 ${ }^{[66]}$, 根据粗细肌丝的空间结构, 构建有负载力 作用情况下分子马达相对细肌丝位移变量满足的 Fokker-Planck 方程, 并推导出肌小节主动收缩力学 模型，根据 $[\mathrm{ATP}]$ 与分子马达状态跃迁速率的耦合关 系, 讨论了 [ATP] 和负载力对肌肉主动力以及收缩速 度的影响, 在一定的分子马达数目、横截面积及弹性 系数情况下, 随着 [ATP]增加, 肌肉主动收缩力逐渐 增大; 收缩速度随着负载力的增加而减小, 直到为零; 肌小节收缩速度随着 [ATP]增加而逐渐增大, 而 当[ATP $]$ 饱和后收缩速度不再改变.

从骨骼肌微观的收缩过程来看, 分子马达的运 动由 $\mathrm{Ca}^{2+}$ 调控, 这是由于 $\mathrm{Ca}^{2+}$ 与肌动蛋白丝上 $\mathrm{Tn}$ 结 合使 $\mathrm{Tm}$ 构象变化导致. Alencar 等人 ${ }^{[67]}$ 从 $\mathrm{Ca}^{2+}$ 与 $\mathrm{Tn}$ 结合调控肌球蛋白结合位点的角度来分析肌肉收缩 原理. Stein 等人 ${ }^{[68]}$ 分析了 $\mathrm{Ca}^{2+}$ 浓度与骨骼肌收缩力 之间关系. 考虑到 $\mathrm{Ca}^{2+}$ 与 $\mathrm{Tn}$ 结合激活细肌丝, 肌浆 中 $\mathrm{Ca}^{2+}$ 浓度可以来表征细肌丝激活程度, 从而决定 了参与做功的分子马达数目, 所以可根据 $\mathrm{Ca}^{2+}$ 与 $\mathrm{Tn}$ 结合的动力学过程, 讨论肌浆中 $\mathrm{Ca}^{2+}$ 浓度对分子马 达集体运行过程的影响. 另外, 从骨骼肌激活过程来 看, 动作电位的性质决定了肌浆中的 $\mathrm{Ca}^{2+}$ 浓度. 因此 对于分子马达集体输出特性的研究也包括从控制角
度分析动作电位频率与 $\mathrm{Ca}^{2+}$ 浓度、主动收缩力、收缩 速度之间的关系, 这也是骨骼肌收缩力学模型从微 观到宏观过渡的一个重要方面, 在文献[69]中我们对 相关内容展开了深人分析, 并结合肌小节的串并联 特征构建了骨骼肌收缩动态力学模型.

\section{4 骨骼肌生物电化学驱动与控制原理}

\section{1 骨骼肌生物电化学驱动原理}

骨骼肌的驱动原理是研究肌肉中的运动神经元 如何激活肌纤维, 启动肌小节内分子马达的循环运 作并调控肌纤维的收缩, 这是一个生物电化学作用 过程. 当运动神经元的动作电位到达神经肌肉接头 后, 运动神经元的动作电位将被复现到肌纤维膜 上 $^{[70]}$. 因此肌纤维膜与运动神经元的动作电位反映 了人体的运动意图, 包含了骨骼肌运行的控制信息. 分子马达启动做功冲程的必要条件是 $\mathrm{Ca}^{2+}$ 与细肌丝 上的 $\mathrm{Tn}$ 结合, 在肌小节的正常运作状况下, 肌浆中 的 $[\mathrm{ATP}]$ 基本饱和, 则 $\left[\mathrm{Ca}^{2+}\right]$ 决定了分子马达做功与 否, 因而 $\mathrm{Ca}^{2+}$ 的活动包含了分子马达的驱动信息.

如图 6(a)所示, 骨骼肌收缩的生物学过程可分为: 动作电位沿 $\mathrm{T}$ 管传递- $\mathrm{SR}$ 中 $\mathrm{Ca}^{2+}$ 扩散至胞浆 $-\mathrm{Ca}^{2+}$ 与 $\mathrm{Tn}$ 结合-启动分子马达运作并使肌小节收缩, 此过程 称作“兴奋-收缩偶联”(ECC coupling $)^{[4]}$. 胞浆中的 $\mathrm{Ca}^{2+}$ 通过 $\mathrm{SR}$ 上的 Ca-ATP 酶进行回收 ${ }^{[71]}$ ，肌小节中 的 $\left[\mathrm{Ca}^{2+}\right]$ 始终处于一个动态平衡状态, 而在肌纤维正 常工作时, SR 的 $\mathrm{Ca}^{2+}$ 通道与 Ca-ATP 酶起主导作用. 如上文所述, 分子马达做功的轨道数目表征了骨骼 肌纤维的激活程度, 也决定了肌纤维的做功功率, 而 激活程度则由胞浆中的 $\left[\mathrm{Ca}^{2+}\right]$ 表征; 因此, 对骨骼肌 的驱动原理研究而言, 关键在于对兴奋-收缩偶联机 制进一步探索并做出理论建模与描述. 尹长城等 人 ${ }^{[72]}$ 通过分析发现 SR 上钙离子通道之间存在物理偶 联，即胞膜的去极化与复极化可使该通道迅速大量 开启或关闭，因而印证了 $\left[\mathrm{Ca}^{2+}\right]$ 调控的源头是动作电 位. Stern 等人 ${ }^{[73]}$ 基于肌膜的生理结构, 对肌小节中 的兴奋-收缩偶联进行了物理建模. Cannell 等人 ${ }^{[74]}$ 对 青蛙肌小节中的 $\mathrm{Ca}^{2+}$ 迁移进行了计算仿真, 只是其 模型忽略了肌膜的具体生理特性, Stuyvers 等人 ${ }^{[75]}$ 考 察了动作电位频率与 $\left[\mathrm{Ca}^{2+}\right]$ 间的稳态关系.

目前对于肌小节驱动特性的研究多针对肌 浆 $\left[\mathrm{Ca}^{2+}\right]$ 的稳态特性或动作电位频率与 $\left[\mathrm{Ca}^{2+}\right]$ 的稳态 
(a) 运动

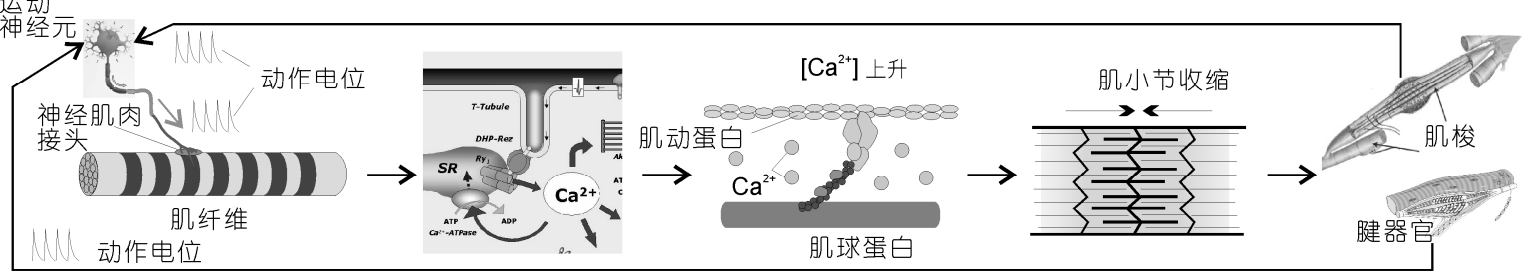

(b)

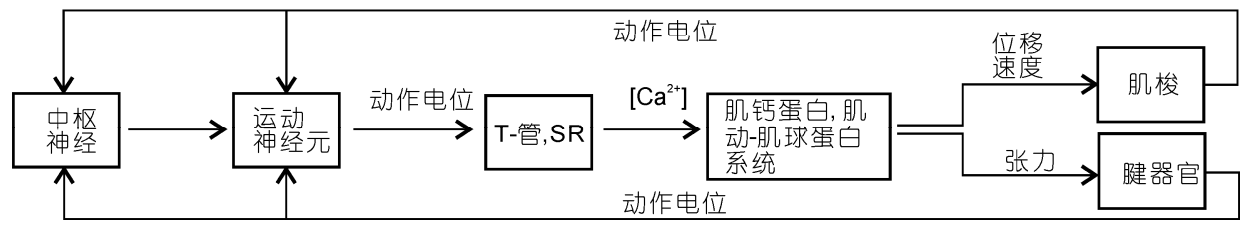

图 6 (a) 骨骼肌收缩的生物学流程; (b) 骨骼肌收缩的控制学流程

关系, 而未涉及 $\left[\mathrm{Ca}^{2+}\right]$ 如何动态变化与传递, 及其如 何被动作电位动态调控. 另一方面, 实验表明肌膜动 作电位的激发频率与肌肉等长收缩力存在正相关关 系 ${ }^{[76]}$, 这表明动作电位的频率与肌小节内的 $\left[\mathrm{Ca}^{2+}\right]$ 也 存在类似关系, 换言之, 动作电位的频率调控着肌纤 维中的 $\left[\mathrm{Ca}^{2+}\right]$. 在文献[77]中, 我们试图对现存的问 题作出改进, 通过开展相关实验, 我们对动作电位如 何在肌膜上形成以及怎样动态调控肌小节中 $\left[\mathrm{Ca}^{2+}\right]$ 建 立了控制学模型, 提出了骨骼肌收缩的生物电化学 变频调控原理. 然而, 目前我们未考虑本体感受器的 反馈作用, 只对骨骼肌收缩过程的开环过程进行建 模, 开环系统存在准确度不高的问题, 难以体现人体 的真实运动意图, 因此很有必要进一步研究骨骼肌 闭环控制原理.

\section{2 骨骼肌收缩的控制原理}

从控制理论角度，骨骼肌的收缩过程是典型的 闭环控制过程, 人体运动是由躯体神经系统所控制. 神经信号以动作电位的形式从中枢神经向下经由皮 质脊髓束, 传递到运动神经元控制肌纤维收缩, 其控 制过程如图 6(b)所示; 因此动作电位是人体运动意图 的信息载体, 分子马达的控制信息即蕴含在运动神 经元所发放的动作电位中. 最早对动作电位进行完 整数学描述的是 Hodgkin 与 Huxley, 他们以枪乌贼巨 轴突为对象 ${ }^{[78]}$, 通过电压钳实验, 对胞膜的 $\mathrm{Na}^{+}$电 流、 $\mathrm{K}^{+}$电流以及泄露电流进行了建模, 并总结了胞膜 的电学模型. 此后随着计算机技术的发展, 更为精细 的模型逐步被提出. Luscher 等人 ${ }^{[79,80]}$ 在已有抽象模 型的基础上, 分别基于等效电路方法与实验研究, 考
查了神经元分叉结构对动作电位传递特性的影响以 及轴突几何尺寸对动作电位传导速度与频率的影响. Rogers 等人 ${ }^{[81]}$ 利用有限元方法对心肌上的动作电位 传递特性进行了建模仿真, 研究了肌纤维的不均匀 性与各向异性对动作电位传递的减速作用, 包括动 作电位波前曲率产生的单向阻滞效应.

如图 6(b)所示, 人体运动的本体感觉是通过动作 电位反馈给中枢神经及运动神经元. 本体感受器是 骨骼肌运动信息检测的硬件基础, 通过感知肌肉收 缩速度、收缩力以及拉伸长度的变化, 向神经中枢提 供信息, 实现骨骼肌的协调控制. 肌肉的本体感受器 主要包括肌梭(muscle spindle)与腱器官(tendon organ) (图 6(a)), 这两种组织分别相当于肌肉的位移/速度及 力传感器. 在肌纤维等长收缩状态下, 腱器官所发放 的动作电位频率不变, 而当肌肉发生被动牵拉时, 其 频率增加. 对同一运动单元的运动神经元来说, 腱器 官信息是负反馈信号, 即抑制收缩; 而肌梭信息是增 强收缩的正反馈信号.

由此可见, 生物神经网络是运动信息反馈通路 及中枢控制单元 ${ }^{[82]}$, 而其中的基本单位是运动神经 元, 因此考察运动神经元如何接受突触的反馈输人、 如何进行信息处理并调节输出就显得十分重要. 研 究表明, 离子通道的分布密度与几何形式对动作电 位的幅值、传导速度与频率都有影响, 且这些物理量 相互耦合. 此外, 由于动作电位懪发的“全或无”特性, 动作电位的频率对分子马达的控制信息尤为关键, 因此需要找出哪些因素/物理量对频率造成影响, 并 研究动作电位频率信息的反馈融合原理, 这关系到 肌梭/腱器官等组织反馈的动作电位如何被运动神经 
元调制并使系统稳定, 最终实现骨骼肌收缩的动态 调控. 另一方面, 目前对于生物神经网络如何根据本 体感知等反馈实现自适应学习仍然了解甚少, 因此 模仿生物神经网络构建具有自适应能力的运动控制 器仍然十分困难，相信这也将是今后骨骼肌控制原 理研究的重点.

\section{5 总结与展望}

基于以上讨论, 可见骨骼肌生物力学原理研究 主要是通过揭示骨骼肌收缩微观机理、驱动与控制原 理, 建立相应的力学和控制系统模型, 探讨模型在仿 生学、生物力学等学科中的应用研究, 针对目前存在 的困难, 存在以下需深人研究的方向.

(1) 分子马达力学原理及运行机制. 研究分子马 达循环过程中的动态力学行为, 探索分子马达在多 种力耦合作用下运行规律, 结合 $(\mathrm{QM} / \mathrm{MM})$ 组合分析 方法, 揭示 ATP 水解后化学能转换为机械能的循环 周期中肌球蛋白的动力学特性, 将分子构象变化与 布朗棘轮机制相结合, 提出更为完善的新理论来解 释分子马达的循环运行机制.

(2) 分子马达力学特性的实验分析. 实验是揭示 分子马达运行规律的必要手段, 可利用 AFM、光镊、 光钳等皮牛级 $(\mathrm{pN})$ 的精密设备, 设计合理的实验方 案, 检测分子马达与细肌丝之间的作用力, 揭示分子 马达多力耦合作用原理及运动机制.

(3) 分子马达集体运行机制研究. 利用非平衡态 统计力学, 分析分子马达循环过程中各个化学状态
与力学特性之间的耦合关系, 构建马达系统的动力 学方程, 在肌小节或肌原纤维层次, 构建新型微力检 测实验平台, 开展分子马达集体力学特性实验验证.

(4) 骨骼肌收缩生物力学模型. 建立动作电位频 率与肌肉收缩力、收缩速度之间的关系, 针对肌小节 的串联与并联特征，建立基于分子马达集体特性的 骨骼肌收缩力学模型. 结合骨骼肌力学模型研究策 略，利用人体宏观层次的实验设备，采集关节作用力 (力矩)及人体生理信号(sEMG 信号)等信息, 开展人 体关节动力学分析, 验证建立在微观力学性质基础 上的骨骼肌力学模型.

(5) 骨骼肌收缩的控制学模型. 明确骨骼肌驱动 及控制环节的物理基础, 即动作电位如何调控肌纤 维的 $\mathrm{Ca}^{2+}$ 浓度, 在此基础上建立相应的物理模型, 并 进一步得到驱动过程的控制学模型, 完善从动作电 位到 $\mathrm{Ca}^{2+}$ 浓度, 从 $\mathrm{Ca}^{2+}$ 浓度到肌肉收缩力的传递函 数. 对于完整的骨骼肌闭环控制模型来说, 需要研究 肌梭与腱器官的收缩速度/收缩力等反馈信息的融合 与控制稳定机制, 并设计相关实验加以验证, 以此来 探索模型应用研究, 如在仿生学上可指导人工肌肉 的仿生设计与控制.

综上所述, 骨骼肌生物力学原理是一项极具挑 战性的研究, 其内容较多, 涉及面较广, 跨度较大, 但是由于其潜在的理论意义和应用价值，已经吸引 了很多研究人员在此展开探索, 而随着新方法和新 技术的应用，我们相信，会有更多规律被揭示，必将 对人体运动机理探索、康复工程等领域产生深远影响.

\section{参考文献}

1 Hill A V. The heat of shortening and the dynamic constants of muscle. Proc R Soc Lond B, 1938, 126: 136-195

2 Huxley H E, Hanson J. Changes in the cross-striations of muscle during contractions and stretch and their structural interpretation. Nature, 1954, 173: 973-976

3 Huxley A F, Niedergerke R. Structural changes in muscle during contraction. Nature, 1954, 173: 971-973

4 余志斌, 李全, 徐彭涛, 等译. 骨骼肌结构与功能. 西安: 第四军医大学出版社, 2010

5 李永胜，陈维毅. 骨骼肌收缩的本构模型: I 被动行为. 力学进展, 2010, 40: 663-678

6 Linke W A, Ivemeyer M, Mundel P, et al. Nature of PEVK-titin elasticity in skeletal muscle. Proc Natl Acad Sci USA, 1998, 95: 8052-8057

7 Rayment I, Holden H M, Whittaker M. Structure of the actin-myosin complex and its implications for muscle contraction. Science, 1993 , 261: 56-65

8 Rayment I, Rypniewski W R, Schmidt-Base K, et al. Three dimensional structure of myosin subfragment-1: A molecular motor. Science, 1993, 261: 50-58

9 Uyeda T Q, Abramson P D, Spudich J A. The neck region of the myosin motor domain acts as a lever arm to generate movement. Proc Natl Acad Sci USA, 1996, 93: 4459-4464 
10 Holmes K C, Angert I, Jon Kull F, et al. Electron cryo-microscopy shows how strong binding of myosin to actin releases nucleotide. Nature, 2003, 425: 423-427

11 Fung Y C. Biomechanics: Mechanical Properties of Living Tissues. New York: Springer-Verlag, 1993. 568

12 李永胜, 张全有, 陈维毅. 骨骼肌收缩的本构模型. 太原理工大学学报, 2005, 36: 760-764

13 Zajac F E. Muscle and tendon: Properties, models, scaling, and application to biomechanics and motor control. Crit Rev Biomed Eng, 1989, 17: 359-411

14 Huxley H E. The mechanism of muscular contraction. Science, 1969, 164: 1356-1366

15 Huxley A F. Muscle structure and theories of contraction. Prog Biophys Biophys Chem, 1957, 7: 255-318

16 Huxley A F, Simmons R M. Proposed mechanism of force generation in striated muscle. Nature, 1971, 233: 533-538

17 Gordon A M, Huxley A F, Julian F J. The variation in isometric tension with sarcomere length in vertebrate muscle fibres. J Physiol, 1966, 184: $170-192$

18 Zahalak G I, Motabarzadeh I. A re-examination of calcium activation in Huxley cross-bridge model. J Biomech Eng, 1997, 119: 20-29

19 Piazzesi G, Reconditi M, Linari M, et al. Mechanism of force generation by myosin heads in skeletal muscle. Nature, 2002, 415: 659-662

20 Finer J T, Simmons R M, Spudich J A. Single myosin molecule mechanics: Pico Newton forces and nano metre steps. Nature, 1994, 368: $113-119$

21 Anderson F C, Pandy M G. Static and dynamic optimization solutions for gait are practically equivalent. J Biomech, 2001, 34: 153-161

22 Neptune R R, Burnfield J M, Mulroy S J. The neuromuscular demands of toe walking: A forward dynamics simulation analysis. J Biomech, 2007, 40: 1293-1300

23 杨义勇, 王人成, 王延利, 等. 含神经控制的下肢肌骨系统正向动力学分析. 清华大学学报(自然科学版), 2006, 46: 1872-1875

24 舒咬根，欧阳钟灿. 生物分子马达. 物理, 2007, 36: 735-741

25 Yamakita Y, Iio T. Conformational change of skeletal muscle troponin. J Biochem, 1989, 105: 870-874

26 Spudich J A. The myosin swinging cross-bridge model. Nat Rev Mol Cell Biol, 2001, 2: 387-392

27 Lymn R W, Taylor E W. Mechanism of adenosine triphosphate hydrolysis by actomyosin. Biochemistry, 1971, 10: 4617-4624

28 赵亚溥. 纳电子机械系统中的若干物理力学问题. 中国力学文摘, 2007, 21: 1-21

29 Munday J N, Capasso F, Parsegian V A. Measured long-range repulsive Casimir-Lifshitz forces. Nature, 2009, 457: 170-173

30 Liu Y M, Scolari M, Im W, et al. Protein-protein interactions in actin-myosin binding and structural effects of R405Q mutation: A molecular dynamics study. Proteins: Struct Funct Bioinform, 2006, 64: 156-166

31 Nakajima H, Kunioka Y, Nakano K, et al. Scanning force microscopy of the interaction events between a single molecule of heavy meromyosin and actin. Biochem Biophys Res Commun, 1997, 234: 178-182

32 郭朝，殷跃红. 肌球蛋白分子马达的多力场耦合机理分析. 科学通报, 2010, 55: 2675-2682

33 Guo Z, Yin Y H. Casimir effect on adhesion interaction between myosin molecular motor and actin filament. Inter J Nanosyst, 2010, 3: 9-15

34 Yanagida S, Kitamura K, Tanaka H, et al. Single molecule analysis of the actomyosin motor. Curr Opin Cell Biol, 2000, 12: 20-25

35 Huxley A F. Cross-bridge action: Present views, prospects, and unknowns. J Biomech, 2000, 33: 1189-1195

36 Kaya M, Higuchi H. Nonlinear elasticity and an 8-nm working stroke of single myosin molecules in myofilaments. Science, 2010, 329: 686-689

37 Sellers J R, Veigel C. Direct observation of the myosin-Va power stroke and its reversal. Nat struct Mol Biol, 2010, 17: 590-595

38 Uyeda T Q P, Abramson P D, Spudich J A. The neck region of the myosin motor domain acts as a lever arm to generate movement. Proc Natl Acad Sci USA, 1996, 93: 4459-4464

39 Ishijima A, Kojima H, Funatsu T, et al. Simultaneous observation of individual ATPase and mechanical events by a single myosin molecule during interaction with actin. Cell, 1998, 92: 161-171

40 Yanagida T, Iwaki M, Ishii Y. Single molecule measurements and molecular motors. Phil Trans R Soc B, 2008, 363: 2123-2134

41 Li G H, Cui Q. Mechanochemical coupling in myosin: A theoretical analysis with molecular dynamics and combined QM/MM reaction path calculations. J Phys Chem B, 2004, 108: 3342-3357

42 Yang Z, Zhao Y P. QM/MM and classical molecular dynamics simulation of His-tagged peptide immobilization on nickel surface. Mat Sci Eng A-Struct, 2006, 423: 84-91

43 Yang Z, Zhao Y P. Adsorption of his-tagged peptide to Ni, Cu and Au (100) surfaces: Molecular dynamics simulation. Eng Anal Bound Elem, 2007, 31: 402-409

44 Feynman R P, Leighton R B, Sands M. The Feynman Lectures on Physics. Boston: Addison-Wesley Longman, 1970, Chap 46

45 Astumian R D. Thermodynamics and kinetics of a Brownian motor. Science, 1997, 276: 917-922

46 Julicher F, Ajdari A, Prost J. Modeling molecular motors. Rev Mod Phys, 1997, 69: 1269-1281

47 Esaki S, Ishii Y, Yanagida T. Model describing the biased Brownian movement of myosin. Proc Jpn Acad, 2003, 79: 9-14 


\section{Biomed Microdevices, 2006, 8: 201-208}

59 Chin L, Yue P, Feng J J, et al. Mathematical simulation of muscle cross-bridge cycle and force-velocity relationship. Biophys J, 2006, 91: 3653-3663

60 郭维生, 罗辽复. 肌球蛋白工作循环的一个新模型. 生物化学与生物物理进展, 2003, 30: 216-220

61 Shu Y G, Shi H L. Cooperative effects on the kinetics of ATP hydrolysis in collective molecular motors. Phys Rev E, 2004, 69: 021912

64 Brugues J, Casademunt J. Self-organization and cooperativity of weakly coupled molecular motors under unequal loading. Phys Rev Lett, 2009, 102: 118104

65 Campas O, Kafri Y, Zeldovich K B, et al. Collective dynamics of interacting molecular motors. Phys Rev Lett, 2006, 97: 038101

66 殷跃红, 郭朝. 分子马达集体运行机制及肌小节动态力学模型. 中国科学: 技术科学, 2011,41: 1533-1540

67 Alencar A M, Butler J P, Mijailovich S M. Thermodynamic origin of cooperativity in acto-myosin interactions: The coupling of short-range interactions with actin bending stiffness in an Ising-like model. Phys Rev E, 2009, 79: 041906

68 Stein R B, Bobet J, Owuztoreli M N, el al. The kinetics relating calcium and force in skeletal muscle. Biophys J, 1988, 54: 705-717

69 郭朝, 殷跃红. 基于分子马达集体运行机制的骨骼肌收缩动态力学模型——基于分子马达运行机制的骨骼肌生物力学原理(I). 中 国科学：技术科学, 2012, 42: 672-679

70 Sanes J R, Lichtman J W. Development of the vertebrate neuromuscular junction. Ann Rev Neurosci, 1999, 22: 389-442

71 Toyoshima C, Nakasako M, Nomura H, et al. Crystal structure of the calcium pump of sarcoplasmic reticulum at $2.6 \AA$ resolution. Nature, 2000, 405: 647-655

72 Yin C C, D'Cruz L G, Lai F A. Ryanodine receptor arrays: Not just a pretty pattern? Cell, 2008, 18: 149-156

73 Stern M D, Pizzaro G, Rios E. Local control model of excitation-contraction coupling in skeletal muscle. J Gen Physiol, 1997, 110: 415-440

74 Cannel M B, Allen D G. Model of calcium movements during activation in the sarcomere of frog skeletal muscle. Biophys J, 1984, 45: 913-925

75 Stuyvers B D, McCulloch A D, Guo J, et al. Effect of stimulation rate, sarcomere length and $\mathrm{Ca}^{2+}$ on force generation by mouse cardiac muscle. J Physiol, 2002, 544: 817-830 学, 2012, 42: 901-910

78 Hodgkin A L, Huxley A F. A quantitative description of membrane current and its application to conduction and excitation in nerve. J Physiol, 1952, 117: 500-544

79 Luscher H R, Shiner J S. Simulation of action potential propagation in complex terminal arborizations. Biophys J, 1990, 58: 1389-1399

80 Smith D O. Mechanisms of action potential propagation failure at sites of axon branching in the crayfish. J Physiol, 1980, 301: 243-259

81 Rogers J M, McCulloch A D. A collocation-Galerkin finite element model of cardiac action potential propagation. IEEE Trans Biomed Eng, 1994, 41: 743-757

82 Kandel E R, Schwartz J H, Jessell T M. Principles of Neural Science. 4th ed. New York: Elsevier, 2000 